\title{
Is subdiffusional transport slower than normal?
}

\author{
Igor Goychuk* \\ Institute of Physics, University of Augsburg, Universitätstr. 1, D-86135 Augsburg, Germany
}

(Dated: July 18, 2018)

\begin{abstract}
We consider anomalous non-Markovian transport of Brownian particles in viscoelastic fluid-like media with very large but finite macroscopic viscosity under the influence of a constant force field $F$. The viscoelastic properties of the medium are characterized by a power-law viscoelastic memory kernel which ultra slow decays in time on the time scale $\tau$ of strong viscoelastic correlations. The subdiffusive transport regime emerges transiently for $t<\tau$. However, the transport becomes asymptotically normal for $t \gg \tau$. It is shown that even though transiently the mean displacement and the variance both scale sublinearly, i.e. anomalously slow, in time, $\langle\delta x(t)\rangle \propto F t^{\alpha},\left\langle\delta x^{2}(t)\right\rangle \propto t^{\alpha}$, $0<\alpha<1$, the mean displacement at each instant of time is nevertheless always larger than one obtained for normal transport in a purely viscous medium with the same macroscopic viscosity obtained in the Markovian approximation. This can have profound implications for the subdiffusive transport in biological cells as the notion of "ultra-slowness" can be misleading in the context of anomalous diffusion-limited transport and reaction processes occurring on nano- and mesoscales.
\end{abstract}

PACS numbers: 05.40.-a, 05.10.Gg, 87.16.Uv

\section{INTRODUCTION}

The widespread occurrence of anomalously slow diffusion and transport [1 9] in biological cells is still not commonly appreciated in spite of a growing experimental evidence and support [10 18]. One of the main psychological obstacles on the way to a wider recognition is that ultraslowness seems intuitively be rather obstructive for the corresponding diffusion-limited biochemical reactions, especially if it is caused by divergent mean residence times (MRTs) in trapping domains [1, 2, 4, 6] created by random meshwork of cell's cytoskeleton. From this perspective, the occurrence of subdiffusion might be more associated with physics of dying, rather than with physics of life. Moreover, the bulk of experimental biophysical data is traditionally interpreted in terms of normal diffusion and even modern biophysics textbooks (see e.g. [20, 21]) discuss only normal diffusion, despite appreciating the fact of existence and importance of intracellular molecular crowding which clearly obstructs diffusion via increase of the effective medium's viscosity [22]. The increase of effective viscosity depends also on the size of diffusing particles. So, "for molecules smaller than $1 \mathrm{~nm}$, it's similar to that of water; for particles of diameter $6 \mathrm{~nm}$ (such as a protein of mass $10^{5} \mathrm{~g} / \mathrm{mol}$ ), it's about 3 times that of water. For 50-500 nm particles, it's 30-300 times that of water" 21] (p. 571). In this respect, typical globular proteins are in the range of 2-20 $\mathrm{nm}$ (diameter) [21, 22] and mRNA molecules are about 400-800 $\mathrm{nm}$ in diameter [15]. However, the traditional thinking and prejudges can also be the reasons for overlooking anomalous diffusion and transport regimes (probably mostly transient) as recent experimental work uncovers [10, 14 17, 19]. Moreover, the occurrence of subdiffusion clearly depends not only on the size of single macromolecules, but also on their concentration, i.e. on the degree of molecular crowding
[16, 19].

One of approaches to anomalously slow diffusion and transport is traditionally based on the assumption of divergent MRTs in trapping domains [1, 2, 4 6 ]. Of course, MRT $\langle\tau\rangle$ in any finite spatial domain with linear size $\Delta x$ can never diverge in real life. However, it can largely exceed a characteristic diffusion time, $\tau_{D} \sim$ $\left(L^{2} / \kappa_{\alpha}\right)^{1 / \alpha}$, required to subdiffusionally explore on average, $\left\langle\delta x^{2}(t)\right\rangle \propto \kappa_{\alpha} t^{\alpha}$ with $0<\alpha<1$, a finite volume with linear size $L \gg \Delta x$, where $\kappa_{\alpha}$ is the corresponding subdiffusion coefficient measured in $\mathrm{cm}^{2} / \mathrm{sec}^{\alpha}[23]$. Then, the approximation of infinite MRTs becomes physically justified on the relevant mesoscopic scale $L$. For very large times $t \gg\langle\tau\rangle$, the diffusion becomes normal, $\left\langle\delta x^{2}(t)\right\rangle \propto t$. However, the corresponding spatial scale can largely exceed $L$ and therefore the normal diffusion (and transport) regime can become of a little importance for certain processes in mesoscopic biochemical reactors of living cells, such as e. g. passive transport of mRNA macromolecules or large globular proteins [15, 16], and in turn subdiffusion becomes of profound importance for such processes on mesoscopic scale. The approximation of divergent MRTs features the continuous time random walk (CTRW) approach to subdiffusion [1, 2, 4, 6]. In this case, the position increments can be totally independent. Within the mean-field approximation, the CTRW transport is congruent [4] with jump-like transport in random potentials. Moreover, the overdamped continuous space Markovian Langevin dynamics in spatially varying potentials can be contracted onto such a semi-Markovian CTRW by doing properly spatial coarse-graining [24, 25]. Then, a potential energy disorder can result in anomalous diffusion and transport in agreement with the semiMarkovian CTRW theory as recent work nicely demonstrates [26] (see also paper by Lindenberg et al. 227] in this Special Issue).

Alternatively, subdiffusion can result from the 
medium's viscoelasticity [11, 13, 16, 19, 28 31]. It can be either due to viscoelasticity of the polymer actin meshwork 13, 16, 28, 29, 32, or due to macromolecular crowding in complex fluids 32 as e.g. in cytoplasm of bacterial cells which are lacking static cytoskeleton [16, 22, 30]. Statistical analysis of the experimental single particle diffusional trajectories in bacteria [15] reveals in fact the primarily viscoelastic origin of subdiffusion 33]. A main result of Ref. [33] is that the fractional Brownian motion scenario (see below) is more likely than one based on a semi-Markovian CTRW [34]. The authors of experimental work [31] came also to a similar conclusion. Within this alternative subdiffusional scenario all the moments of random time spent in finite spatial domains remain finite. The corresponding MRT is not only finite but it scales down to zero with $\Delta x \rightarrow 0$. The physical reason for subdiffusion here is very different. It occurs due to long-time anticorrelations in the position increments [30]. Considering Brownian particle of radius $R$, which starts to move at $t_{0}=0$ with velocity $\dot{x}(t)$ (we consider a one-dimensional case for simplicity), one expects it to experience a viscoelastic force

$$
F_{\mathrm{v}-\mathrm{el}}(t)=-\int_{0}^{t} \eta\left(t-t^{\prime}\right) \dot{x}\left(t^{\prime}\right) d t^{\prime}
$$

where $\eta(t)$ is a frictional memory kernel whose Laplacetransform $\tilde{\eta}(s)$ is related to the frequency-dependent medium's viscosity $\tilde{\zeta}(i \omega)$ as $\tilde{\eta}(s)=6 \pi R \tilde{\zeta}(s)$. In the case of purely viscous fluids, and in neglecting the hydrodynamic memory effects, $\zeta(t)=2 \zeta_{0} \delta(t)$, where $\zeta_{0}$ is the fluid's macroscopic viscosity, so that $\eta(t)=2 \eta_{0} \delta(t)$, where $\eta_{0}=6 \pi R \zeta_{0}$ is the Stokes viscous friction coefficient. For weakly viscoelastic fluids, $\zeta(t)=\zeta_{0} \nu \exp (-\nu t)$, exponentially decays in time with rate $\nu$, and correspondingly $\eta(t)=6 \pi R \zeta_{0} \nu \exp (-\nu t)=\kappa \exp (-\nu t)$, where $\kappa$ has dimension of a linear elastic force constant. This corresponds to the Maxwell theory of viscoelasticity [35] who derived the phenomenon of viscosity from medium's elasticity by assuming that the linear elastic force, $F_{\mathrm{el}}(t)=$ $-\kappa[x(t)-x(0)]$, acting on the particle can relax in time with rate $\nu$, yielding a viscoelastic force, i.e. $\dot{F}_{\mathrm{v}-\mathrm{el}}(t)=$ $-\kappa \dot{x}(t)-\nu F_{\mathrm{v}-\mathrm{el}}(t)$. Indeed, if the force relaxation is very fast with respect to the change of particle's velocity, then $F_{\mathrm{v}-\mathrm{el}}(t)=-\int_{0}^{t} \eta\left(t-t^{\prime}\right) \dot{x}\left(t^{\prime}\right) d t^{\prime} \approx-\eta_{0} \dot{x}(t)$, with $\eta_{0}=\kappa / \nu$, whereas in the opposite limit, $F_{\mathrm{v}-\mathrm{el}}(t) \approx$ $-\kappa[x(t)-x(0)]$. For strongly viscoelastic media one expects that $\eta(t)$ decays in time much slower than exponential and a power law decay $\eta(t) \propto t^{-\alpha}$ can serve as a better model. For a fluid-like environment the effective macroscopic viscosity $\zeta_{0}=\int_{0}^{\infty} \zeta(t) d t$ should remain, however, finite. It can be very large, but yet finite. Therefore, a long-time cutoff to the power-law must exist. In 1936, A. Gemant proposed a class of power-law viscoelastic models which are consistent with this demand [36, 37]. Its particular representative corresponds in the
Laplace space to

$$
\tilde{\eta}(s)=\int_{0}^{\infty} \eta(t) \exp (-s t) d t=\frac{\eta}{1+(s \tau)^{1-\alpha}},
$$

in our notations. Here, $\tilde{\eta}(0)=\eta$ is an effective asymptotic friction coefficient and $\tau$ presents a long-time memory cutoff. The corresponding memory kernel is approximately

$$
\eta(t) \approx \frac{\eta_{\alpha}}{\Gamma(1-\alpha) t^{\alpha}}
$$

for $t \ll \tau$, where $\eta_{\alpha}=\eta \tau^{\alpha-1}$, and $\Gamma(x)$ is the familiar gamma-function. For $t \gg \tau, \eta(t)$ decays also in accordance with a power law, $\eta(t) \propto t^{\alpha-2}$, i.e. elastic correlations are still rather strong. However, the corresponding integral converges ensuring that the asymptotic friction coefficient $\eta$ is finite. In the limit of infinitely large medium's viscosity yielding $\eta \rightarrow \infty$, and infinitely long memory range, $\tau \rightarrow \infty$, with $\eta_{\alpha}=\eta \tau^{\alpha-1}$ kept constant, Eq. (3) becomes exact, $\tilde{\eta}(s)=\eta_{\alpha} s^{\alpha-1}$, and

$$
\begin{aligned}
F_{\mathrm{v}-\mathrm{el}}(t) & =-\int_{0}^{t} \frac{\eta_{\alpha}}{\Gamma(1-\alpha)\left(t-t^{\prime}\right)^{\alpha}} \dot{x}\left(t^{\prime}\right) d t^{\prime} \\
& :=-\eta_{\alpha 0} D_{*}^{\alpha} x(t),
\end{aligned}
$$

where the last equality defines fractional Caputo derivative of the order $0<\alpha<1$ [38] acting on $x(t)$. $\eta_{\alpha}$ can be named the fractional friction coefficient. Clearly, Eqs. (314) can serve as a good approximation only for the times $t \ll \tau$. In the focus of this Letter is but the entire time evolution, interpolating between transient subdiffusion and asymptotically normal diffusion behavior. For example, $\tau$ can correspond to the time scale of seconds or minutes, and then subdiffusion emerges on the time scale from microseconds to seconds or minutes, as in biological cells 15 17, 19].

\section{SIMPLE MODEL}

We continue with a non-Markovian generalized Langevin equation (GLE) description [39 41] for an overdamped Brownian particle neglecting inertial effects. Then,

$$
\int_{0}^{t} \eta\left(t-t^{\prime}\right) \dot{x}\left(t^{\prime}\right) d t^{\prime}=f(x, t)+\xi(t),
$$

where $f(x, t)$ is a generally nonlinear force acting on the particle and $\xi(t)$ is a thermal random force of the environment. It is Gaussian, unbiased on average, and obeying the fluctuation-dissipation relation,

$$
\left\langle\xi(t) \xi\left(t^{\prime}\right)\right\rangle=k_{B} T \eta\left(\left|t-t^{\prime}\right|\right),
$$

at the environmental temperature $T$. This is required for the consistency with thermodynamics at thermal equilibrium. In the above-mentioned limit $\tau \rightarrow \infty, \eta \rightarrow$ 


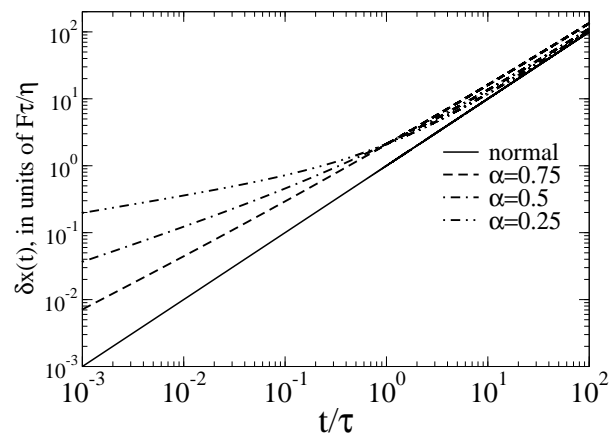

FIG. 1. Mean displacement (in units of $F \tau / \eta$ ) versus time (in units of $\tau$ ) under the influence of constant force $F$ for several different values of $\alpha$ and the same $\eta, \tau$. The limit of normal diffusion is achieved asymptotically from above. Transiently subdiffusing particles always cover larger distances than normally diffusing particles with the same asymptotic frictional force constant $\eta$.

$\infty, \eta_{\alpha}=$ const, GLE (5) is named also the fractional Langevin equation [9] upon the use of the corresponding abbreviation (4) for its lhs. In this limit, $\xi(t)$ is nothing else but the fractional Gaussian noise by Mandelbrot and van Ness [42] which presents an instance of $1 / f$ noise with the spectral power density $S(\omega) \propto 1 / \omega^{1-\alpha}$. Notice that generally the lower integration limit in Eq. (5) is $t_{0} \rightarrow-\infty$. It can be replaced, however, with $t_{0}=0$ since we assume that the particle starts to move at this time being initially localized, i.e. $v(t)=\dot{x}(t)=0$ for $t<t_{0}$.

Let us consider the transport under the influence of constant force $F$. Then, the above GLE can be easily solved formally using the Laplace-transform method, for any memory kernel $\eta(t)$. Transforming back to the time domain for the memory kernel (2), one obtains for the averaged mean displacement the simple result,

$$
\begin{aligned}
\langle\delta x(t)\rangle & =\frac{F t}{\eta}+\frac{F t^{\alpha}}{\eta_{\alpha} \Gamma(1+\alpha)} \\
& =\frac{F \tau}{\eta}\left[\frac{t}{\tau}+\frac{1}{\Gamma(1+\alpha)}\left(\frac{t}{\tau}\right)^{\alpha}\right] .
\end{aligned}
$$

This exact solution is compared in Fig 1 with the solution of the ordinary Langevin equation with memoryless friction for the particle which experiences the same frictional force for the whole time span as our particle asymptotically, or, said differently, the result of the Markovian approximation to the considered dynamics. Clearly, for all times our particle moves in fact faster, covering larger distances and approaching gradually the limit of normal diffusion from above. The instant time-dependent ensemble-averaged velocity $\langle v(t)\rangle:=\langle\delta \dot{x}(t)\rangle=F / \eta+$ $F /\left[\eta_{\alpha} \Gamma(\alpha) t^{1-\alpha}\right]$ is also always larger than its asymptotic value $F / \eta$ (a spurious singularity at $t=0$ can be eliminated, if to take the initial inertial effects into account). Mathematically, this is simply because $x^{\alpha} \gg x$, for $x \ll 1$ and $0<\alpha<1$. The physics is also clear. In the limit $\eta \propto \zeta_{0} \rightarrow \infty$ the normally diffusing particle is get localized, $\langle\delta x(t)\rangle \rightarrow 0$, whereas our particle still moves, but ultra slow (per definition) since $\langle\delta x(t)\rangle \propto(t / \tau)^{\alpha}$. Therefore, the "ultra-slow" moving particle can cover larger distances. Furthermore, for any memory kernel in the studied model and for arbitrary constant $F$ the variance of the particle position, $\left\langle\delta x^{2}(t)\right\rangle=\left\langle x^{2}(t)\right\rangle-\langle x(t)\rangle^{2}$ obeys [39]

$$
\left\langle\delta x^{2}(t)\right\rangle=\frac{2 k_{B} T}{F}\langle\delta x(t)\rangle,
$$

and therefore it follows to the same pattern as in Eq. (7) and Fig. 1,

$$
\left\langle\delta x^{2}(t)\right\rangle=2 \kappa_{1} t+2 \kappa_{\alpha} t^{\alpha} / \Gamma(1+\alpha) .
$$

Here, $\kappa_{\alpha}$ is fractional diffusion coefficient related to temperature and fractional friction coefficient by the generalized Einstein-Stokes relation, $\kappa_{\alpha}=k_{B} T / \eta_{\alpha}$, which contains the standard one, $\kappa_{1}=k_{B} T / \eta$, as a particular case.

We suppose that our observation is rather general. For example, the results in Ref. [15] seem to agree with our line of reasoning. Indeed, mRNA macromolecules have in the related experiments radii in the range of 200-500 nm. Furthermore, the normal diffusion coefficient in water was found to be $\kappa_{1}=1 \mu \mathrm{m}^{2} / \mathrm{sec}$ (see Supplementary Material in [15]). From this, given the water viscosity $\zeta_{w}=0.9 \cdot 10^{-3} \mathrm{~Pa} \cdot \mathrm{sec}$, one can estimate the corresponding radius as $R=k_{B} T /\left(6 \pi \zeta_{w} \kappa_{1}\right)$ which gives $R \approx 242 \mathrm{~nm}$ for $T=300 \mathrm{~K}$. Let us assume that $R \approx 250 \mathrm{~nm}$. Then, the corresponding macroscopic normal diffusion coefficient in cytosol should be by the factor of $r \approx 300$ smaller than one in water 21] (see the above quotation in Introduction). This yields $\kappa_{1}^{(c y t)} \approx \kappa_{1} / r \approx 3.33 \cdot 10^{-3} \mu \mathrm{m}^{2} / \mathrm{sec}$. However, the experiment yields not normal but subdiffusion with $\alpha \approx 0.7$ (see Fig. 2(a) in [15]) and $\kappa_{\alpha}$ in the range from $10^{-3}$ to $10^{-2} \mu \mathrm{m}^{2} / \mathrm{sec}^{0.7}$ [34]. Assuming $\kappa_{\alpha}=10^{-2} \mu \mathrm{m}^{2} / \mathrm{sec}^{0.7}$ for this value of $R$ (smaller particles in experiment should also subdiffuse faster) one can conclude that subdiffusion can indeed cover larger distances than normal diffusion with $\kappa_{1}^{(c y t)} \sim \kappa_{1} / r$. Furthermore, one can estimate the transition time $\tau$. Given the relation $\tau=\left(\eta / \eta_{\alpha}\right)^{1 /(1-\alpha)}=\left(\kappa_{\alpha} / \kappa_{1}^{(c y t)}\right)^{1 /(1-\alpha)}$ which follows within our model one obtains for it $\tau \approx 55 \mathrm{sec}$. This is a rather reasonable estimate since subdiffusion regime lasts in those experiments up to $30 \mathrm{sec}$, cf. Fig. $2(\mathrm{a})$ in [15].

\section{CONCLUSIONS}

The discussed phenomenon might seem paradoxical, even though its explanation is almost trivial. Nevertheless, it has profound implications for subdiffusion in biological cells. First of all, the occurrence of subdiffusion 
on some transient time scale $\tau$ and the corresponding mesoscopic spatial scale $L \sim\left(2 \kappa_{\alpha} \tau^{\alpha}\right)^{1 / 2}$ does not contradict to the bulk of macroscopic experimental data indicating typically a normal diffusion [21]. Even more important, the overall transport is in fact faster than its long time normal asymptotics that results from a drastic reduction of the effective normal diffusion coefficient for large macromolecules due to molecular crowding effects in cytosol. Very important is also the fact that on the time scale $t \ll \tau$, by use of the memory kernel (4) as approximation, the resulting random process is nothing else the fractional Brownian motion (fBm) [30, 42]. The fractal dimensionality of the $\mathrm{fBm}$ trajectories is known to be $d_{f}=2 / \alpha$ [43], i.e. $d_{f}=2$ for the normal Brownian motion. However, for $\alpha \leq 2 / 3$ and for $3 \mathrm{~d}$ Euclidean embedding dimension it becomes $d_{f}=3$ (a fractal trajectory cannot have larger Hausdorff dimension that the Euclidean space in which it is living). This means that a subdiffusionally searching particle explores much more thoroughly finite volumes than a normally diffusing particle on the same spatial and time scales. Therefore, transient subdiffusion can provide only advantages for the diffusion-limited reactions in tiny biochemical reactors of living cells, which are densely stuffed with different macromolecules, without some principal drawbacks. This is rather unexpected and paradoxical conclusion.

\section{ACKNOWLEDGMENTS}

This paper is dedicated to Professor P.V.E. McClintock on occasion of his 70th birthday. Support of this work by the Deutsche Forschungsgemeinschaft, grant GO 2052/1-1 is gratefully acknowledged.

igor.goychuk@physik.uni-augsburg.de

[1] M. F. Shlesinger, Asymptotic solutions of continuous time random walks, J. Stat. Phys. 10 (1974) 421-434.

[2] H. Scher and E. M. Montroll, Anomalous transit time dispersion in amorphous solids, Phys. Rev. B 12 (1975) 2455-2477.

[3] J.-P. Bouchaud and A. Georges, Anomalous diffusion in disordered media: statistical mechanisms, models and physical applications, Phys. Rep. 195 (1990) 127-293.

[4] B. D. Hughes, Random walks and Random Environments, Vols. 1,2 (Clarendon Press, Oxford, 1995).

[5] M. F. Shlesinger, Random processes, in: Encyclopedia of Applied Physics 16 (VCH Publishers, 1996), pp. 45-70.

[6] R. Metzler and J. Klafter, The random walk's guide to anomalous diffusion: a fractional dynamics approach, Phys. Rep. 339 (2000) 1-77.

[7] R. Balescu, Statistical dynamics: matter out of equilibrium (Imperial College Press, London, 1997).

[8] Anomalous Transport: Foundations and Applications, R. Klages, G. Radons, and I. M. Sokolov (Eds.) (WileyVCH, Weinheim, 2007).
[9] Fractional Dynamics: Recent Advances, R. Metzler, S. C. Lim, and J. Klafter (Eds.) (World Scientific, Singapore, 2011).

[10] M. Wachsmuth, W. Waldeck, and J. Langowski, Anomalous diffusion of fluorescent probes inside living cell nuclei investigated by spatially-resolved fluorescence correlation spectroscopy, J. Mol. Biol. 298 (2000) 677-689.

[11] H. Qian, Single-particle tracking: Brownian dynamics of viscoelastic materials, Biophys. J. 79 (2000) 137-143.

[12] G. Seisenberger, et al., Real-time single-molecule imaging of the infection pathway of an adeno-associated virus, Science 294 (2001) 1929-1932.

[13] A. Caspi, R. Granek, and M. Elbaum, Phys. Rev. E 66 (2002) 011916.

[14] I. M. Tolić-Nørrelykke, et al., Anomalous diffusion in living yeast cells, Phys. Rev. Lett. 93 (2004) 078102.

[15] I. Golding and E. C. Cox, Physical nature of bacterial cytoplasm, Phys. Rev. Lett. 96 (2006) 098102.

[16] G. Guigas, C. Kalla, and M. Weiss, Probing the nanoscale viscoelasticity of intracellular fluids in living cells, Biophys. J. 93, (2007) 316-323.

[17] S. C. Weber, A. J. Spakowitz, and J. A. Theriot, Bacterial chromosomal loci move subdiffusively through a viscoelastic cytoplasm, Phys. Rev. Lett. 104 (2010) 238102.

[18] H. Sheng, Y. Q. Chen, and T. Qiu, Heavy-tailed distribution and local long memory in time series of molecular motion on the cell membrane, Fluct. Noise Lett. 10 (2011) 93-119.

[19] W. Pen, et al., Viscoelasticity in homogeneous protein solutions, Phys. Rev. Lett. 102 (2009) 058101.

[20] M. B. Jackson, Molecular and Cellular Biophysics (Cambridge University Press, Cambridge, 2006).

[21] P. Nelson, Biological Physics: Energy, Information, Life (Freeman and Co., New York, 2004).

[22] T. D. Pollard, W. C. Earnshow, J. Lippincott-Schwartz, Cell Biology, 2nd. ed. (Sounders Elsevier, Philadelphia, 2008).

[23] I. Goychuk and P. Hänggi, Fractional diffusion modeling of ion channel gating, Phys. Rev. E 70 (2004) 051915.

[24] P. Reimann, C. Van der Broek, H. Linke, P. Hänggi, J. M. Rubi, A. Perez-Madrid, Giant acceleration of free diffusion by use of tilted periodic potentials, Phys. Rev. Lett. 87, 010602 (2001); ibid., Diffusion in tilted periodic potentials: enhancement, universality, and scaling, Phys. Rev. E 65, 031104 (2002).

[25] B. Lindner, M. Kostur, and L. Schimansky-Geier, Optimal diffusive transport in a tilted periodic potential, Fluct. Noise Lett. 1 (2001) R25-R39.

[26] M. Khoury, A. M. Lacasta, J. M. Sancho, and K. Lindenberg, Weak disorder: anomalous transport and diffusion are normal yet again, Phys. Rev. Lett. 106 (2011) 090602.

[27] K. Lindenberg, J. M. Sancho, M. Khoury, and A. M. Lacasta, Weak disorder in periodic potentials: anomalous transport and diffusion, Fluct. Noise Lett. 11 (2012) xxx.

[28] T. G. Mason and D. A. Weitz, Optical measurements of frequency-dependent linear viscoelastic moduli of complex fluids, Phys. Rev. Lett. 74 (1995) 1250-1253.

[29] F. Amblard, et al., Subdiffusion and anomalous local viscoelasticity in actin networks, Phys. Rev. Lett. 77 (1996) 4470-4473.

[30] I. Goychuk, Viscoelastic subdiffusion: from anomalous to normal, Phys. Rev. E 80 (2009) 046125.

[31] J. Szymanski and M. Weiss, Elucidating the origin of anomalous diffusion in crowded fluids, Phys. Rev. Lett. 
103 (2009) 038102.

[32] T. A. Weigh, Microrheology of complex fluids, Rep. Prog. Phys. 68 (2005) 685-742.

[33] M. Magdziarz, A. Weron, K. Burnecki, J. Klafter, Fractional Brownian motion versus the continuous-time random walk: a simple test for subdiffusive dynamics, Phys. Rev. Lett. 103, 180602 (2009).

[34] Visible scattering in the distribution of single trajectories can reflect e.g. distribution of mRNA molecules over their sizes, binding properties, etc. [22]; this being in a combination with the absence of self-averaging even in the case of ergodic diffusion for experimental trajectories of finite length.

[35] J. C. Maxwell, On the dynamical theory of gases, Phil. Trans. R. Soc. Lond. 157 (1867) 49-88.

[36] A. Gemant, A method of analyzing experimental results obtained from elasto-viscous bodies, Physics 7 (1936)
$311-317$.

[37] I. Goychuk, Viscoelastic subdiffusion: generalized Langevin equation approach, Adv. Chem. Phys. 150 (2012) 187-253.

[38] R. Gorenflo and F. Mainardi, in: Fractal and Fractal Calculus in Continuum Mechanics, ed. by A. Carpinteri and F. Mainardi (Springer, Wien, 1997), 223-276.

[39] R. Kubo, The fluctuation-dissipation theorem, Rep. Prog. Phys. 29 (1966) 255-284.

[40] R. Zwanzig, Nonlinear generalized Langevin equations, J. Stat. Phys. 9 (1973) 215-220.

[41] R. Kubo, M. Toda, and M. Hashitsume, Nonequilibrium Statistical Mechanics, 2nd ed. (Springer, Berlin, 1991).

[42] B. B. Mandelbrot and J. W. van Ness, Fractional Brownian motions, fractional noises and applications, SIAM Review 10 (1968) 422-437.

[43] J. Feder, Fractals (Plenum Press, New York, 1991). 